\title{
TWEETS SENTIMENT ON PPKM POLICY AS A COVID-19 RESPONSE IN INDONESIA
}

\author{
Seng Hansun \\ Senior Lecturer and Researcher, Informatics Department, Universitas Multimedia Nusantara, \\ Tangerang, Indonesia \\ seng.hansun@lecturer.umn.ac.id \\ Alethea Suryadibrata \\ Lecturer, Informatics Department, Universitas Multimedia Nusantara, \\ Tangerang, Indonesia \\ alethea@umn.ac.id \\ Rossy Nurhasanah \\ Senior Lecturer, Information Technology Department, Universitas Sumatera Utara, \\ Medan, Indonesia \\ rossynurhasanah@usu.ac.id \\ Jaka Fitra \\ Lecturer, Software Engineering Department, Institut Teknologi dan Bisnis Diniyyah Lampung, \\ Lampung, Indonesia \\ jakafitra@instidla.ac.id
}

\begin{abstract}
The Coronavirus Disease 2019 (COVID-19) has roamed for almost two years now. Every country has applied its strategies in facing and handling this pandemic, including Indonesia. One strategy applied by the Indonesian government in handling this crisis is the enforcement of restrictions on community activities (PPKM) policy. This policy has been acknowledged by many countries' leaders as an effective strategy in handling the COVID-19 pandemic without giving too much burden to the economic sector. However, despite the pros, there are also cons of the policy in society. Therefore, we are interested in conducting a sentiment analysis for the PPKM policy based on Twitter tweets data. We found that most of the tweets were dominated by the neutral sentiment $(58.07 \%)$, followed by the positive sentiment $(27.12 \%)$, and lastly by the negative sentiment (14.81\%). Furthermore, we also try to build a deep learning model based on long short-term memory (LSTM) networks for the classification task of the collected tweets. We found the proposed deep learning model could reach $\mathbf{9 2 . 5 9 \%}$ accuracy on the test set, which is pretty high for this sentiment analysis classification task. The built model then was deployed as a simple web-based application that can be accessed freely in the Heroku platform.
\end{abstract}

Keywords: COVID-19 response, Heroku, Indonesia, LSTM, PPKM policy, sentiment analysis, Twitter.

\section{Introduction}

The Coronavirus Disease 2019 (COVID-19) has roamed for almost two years now. Its first case was detected in late 2019 by the Chinese Government [Acter et al., (2020)], which was later greatly impacted the whole world. The number of confirmed cases has exponentially increased within a short time period, driving the World Health Organization (WHO) to declare it as a pandemic in March 2020 [Spinelli and Pellino, (2020)]. It was recorded that more than 240 million confirmed cases were reported with around 4.9 million deaths worldwide per October 2021 [World Health Organization, (2021)].

In Indonesia, the first multiple COVID-19 cases were officially reported on March $2^{\text {nd }}, 2020$ by President Joko Widodo [Asyary and Veruswati, (2020); Djalante et al., (2020)]. Since then, the country has faced the dynamic changing of the pandemic situation with several national strategies. One of them is the enforcement of restrictions on community activities policy (Pemberlakuan Pembatasan Kegiatan Masyarakat), which later in this paper will be referred to as the PPKM policy. The restrictions are made mainly by regulating activities and operational hours for community activities [Ginting, (2021)]. This policy has replaced the previous policy that took place in the 
early period of the COVID-19 pandemic in 2020 [Muhyiddin and Nugroho, (2021)]. PPKM policy has been placed since January 2021 and has undergone several changes during its implementation according to the pandemic situation and development.

Since the policy has been enforced for ten months, some pros and cons to the policy can be easily found in the community's daily conversation. Some of the pros are the number of COVID-19 confirmed cases has significantly reduced after the policy has been enforced [Toharudin et al., (2021)] and the economic sector has started to restore to its previous state before the crisis happened by balancing the policy with economic activities [Kennedy, (2021)]. Meanwhile, some of the cons are the continuation of PPKM policy, which is evaluated bi-weekly, seems not to end immediately and the impact to get proper income sources had burdened many people, especially the poor ones.

In this study, we are interested to learn and analyzing the community opinions on the PPKM policy. We limit our focus on the conversation in Twitter, one of the top ten social media platforms based on the monthly active users [Walsh, (2021)]. The contributions of this study are 1) we conduct the sentiment analysis on the PPKM policy as a response to the COVID-19 pandemic in Indonesia, 2) we use relevant recent tweets from the Twitter platform, 3) we further analyze and classify the tweets by using a deep learning method, i.e., the long short-term memory (LSTM) networks, 4) using the built model, we develop a simple web-based application that can automatically classify any tweets related to the PPKM policy in Indonesia.

The structure of this paper is as follows. In the following section, the data source and a brief explanation of LSTM networks will be explained. Next, we describe the research methodology and sentiment analysis results. Furthermore, we explain the deep learning model development and the web-based application results. Finally, some concluding remarks will be given at the end of the paper's structure.

\section{Data Source and Method}

We start this section by explaining Twitter tweets as the data source followed by a description of LSTM networks, the deep learning method employed in this study. Moreover, we also give a brief description of several performance metrics reported in this study by using a confusion matrix.

\subsection{Data source}

The main data source used in this study is tweets data from Twitter, a popular social media platform in Indonesia. Twitter provides an Application Programming Interface (API) that enables us to collect tweets based on a particular search query. Twitter API had been utilized in many studies, especially for sentiment analysis research, as can be seen in the works of [Rufai and Bunce (2020); Ribeiro et al. (2018); Fitri et al. (2019); and Suhartono et al. (2017)].

In this study, we utilize Twitter API and several other libraries to access the needed tweets data and perform the sentiment analysis process. Some of those libraries are Tweepy - a Python client that provides direct access to Twitter API, TextBlob - a Python library for textual data processing, and GoogleTrans - a Python library for automatic language translation. TextBlob is the main library used in the sentiment analysis process. It provides common natural language processing tasks, where the default classifier used for sentiment analysis is the Naïve Bayes Classifier [Loria, (2020)]. We also limit the tweets data collection from August $1^{\text {st }}, 2021$ to September 24 $4^{\text {th }}$, 2021 where the results are shown in Table 1.

Table 1. Collected tweets data and sentiment results.

\begin{tabular}{|c|c|c|c|}
\hline \multirow{2}{*}{ Tweets Collected } & \multicolumn{3}{|c|}{ Sentiment Results using TextBlob } \\
\hline & Positive & Negative & Neutral \\
\hline Number & 163 & 89 & 349 \\
\hline Percentage & $27.12 \%$ & $14.81 \%$ & $58.07 \%$ \\
\hline Example & $\begin{array}{c}\text { ppkm efektif menurunkan positif } \\
\text { rate nasional } \\
\text { Bersatu Ikut Vaksinasi } \\
\text { https://t.co/o8sGfsR33n }\end{array}$ & $\begin{array}{l}\text { PPKM di perpanjang trusssss...... } \\
\text { Tambah byk rakyat yg susah mkn } \\
\text { dech }\end{array}$ & $\begin{array}{l}\text { Disaat sudah turun ke Level-3, agar } \\
\text { tetap disiplin protokol kesehatan,. } \\
\text { perkuat pengendalian Covid-19 } \\
\text { dengan 3T (Te... } \\
\text { https://t.co/mRxIcjSQpb }\end{array}$ \\
\hline
\end{tabular}

\subsection{Long Short-Term Memory (LSTM)}

LSTM is a special type of recurrent neural networks (RNNs), which was proposed by Hochreiter and Schmidhuber, to tackle the long-term dependency problem found in conventional RNNs [Yu et al., (2019)]. It introduces a gates mechanism that consists of three gates, namely the forget gate, the input gate, and the output gate. Forget gate is the first gate that can be found in an LSTM cell. It controls the information loss degree from the prior cell's hidden state. Input gate can be found next, which controls the new information degree to be stored in the current cell state. Output gate is the last gate, which is used to determine the new output for the current cell 
[Hansun and Young, (2021)]. Several equations related to the computing process of an LSTM cell are shown as Eq.(1) to Eq.(6).

$$
\begin{gathered}
f_{t}=\sigma\left(W_{f} h_{t-1}+U_{f} x_{t}+b_{f}\right) \\
i_{t}=\sigma\left(W_{i} h_{t-1}+U_{i} x_{t}+b_{i}\right) \\
\tilde{C}_{t}=\tanh \left(W_{C} h_{t-1}+U_{C} x_{t}+b_{C}\right) \\
C_{t}=f_{t} \odot C_{t-1}+i_{t} \odot \tilde{C}_{t} \\
o_{t}=\sigma\left(W_{o} h_{t-1}+U_{o} x_{t}+b_{o}\right) \\
h_{t}=o_{t} \odot \tanh \left(C_{t}\right)
\end{gathered}
$$

$f_{t}, i_{t}$, and $o_{t}$ are the forget, input, and output gate, respectively. $\tilde{C}_{t}$ and $C_{t}$ are the candidate cell and the current cell state. $h_{t}$ and $x_{t}$ are the current hidden state and the new input value. $W_{f}, W_{i}, W_{C}, W_{o}, U_{f}, U_{i}, U_{C}, U_{o}$ are the networks' weights, while $b_{f}, b_{i}, b_{C}, b_{o}$ are bias weights. There are two activation functions used, i.e., sigmoid $(\sigma)$ and tangent hyperbolic (tanh). They are the most frequently used non-linear activation functions in artificial neural networks [Li and Zhao, (2019)]. An illustration of an LSTM cell is shown in Fig.1.
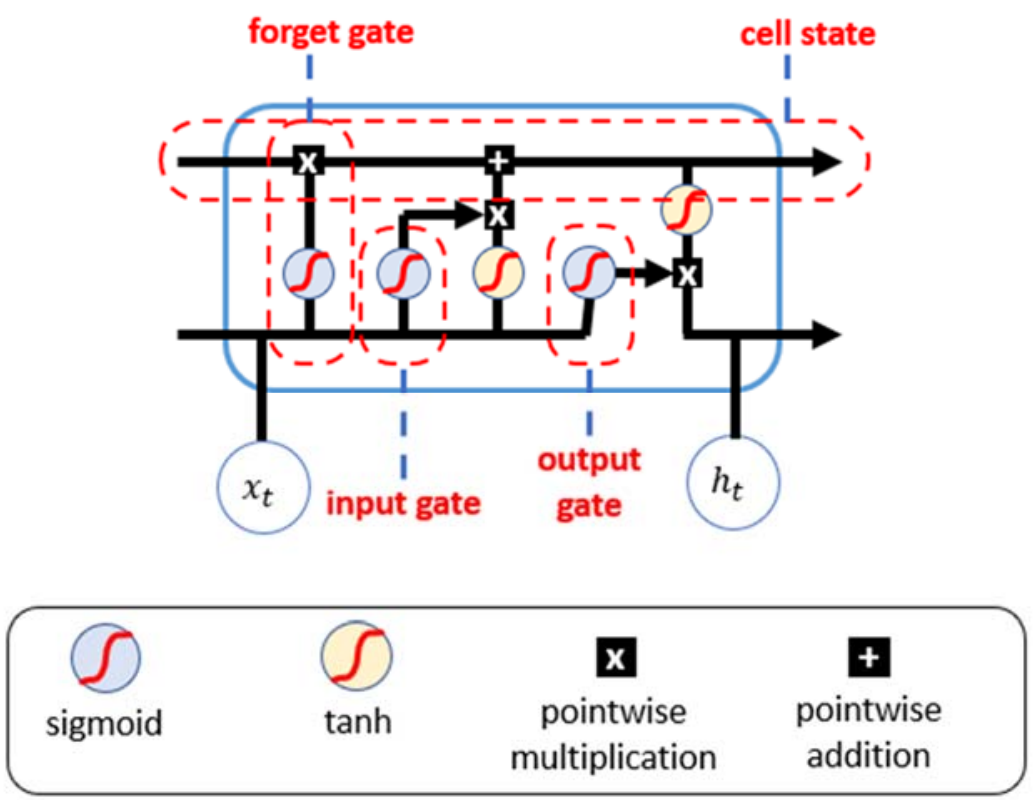

Fig. 1. LSTM cell and its gates mechanism [Hansun and Suryadibrata, (2021)]

\subsection{Confusion matrix}

Confusion matrix refers to a table that shows the amount of both correctly and incorrectly classified data between the actual and predicted classification results [Ferdina et al., (2019)]. Using this matrix, we could compute several metrics that are commonly used in evaluating the performance of a classification method, such as precision, recall, and F1 score. Precision shows the ratio of correctly predicted positive class to the total predicted positive class, while Recall refers to the ratio of correctly predicted positive class to the total actual positive class. F1 is the harmonic mean between precision and recall, which means that both false positives and false negatives are weighted equally [Arora et al., (2019)]. Eq.(7) to Eq.(9) show formulas for calculating precision, recall, and F1 score respectively [Yunita and Hansun, (2019)].

$$
\begin{gathered}
\text { Precision }=\frac{\text { True Positive }}{\text { True Positive }+ \text { False Positive }} \\
\text { Recall }=\frac{\text { True Positive }}{\text { True Positive }+ \text { False Negative }} \\
\text { F1 score }=\frac{2 \times \text { Precision } \times \text { Recall }}{\text { Precision }+ \text { Recall }}
\end{gathered}
$$

\section{Research Methodology}

In this study, we use SEMMA data mining methodology as introduced by SAS Institute [Rahim, et al., (2017)]. There are five steps in SEMMA that form the acronym, namely sample, explore, modify, model, and access [Tariq et al., (2019)]. SEMMA has been adopted widely in various scenarios, such as monitoring of water consumption 
for an irrigation system [López-Torres et al., (2020)], predicting the cricket competition winner [Vistro, et al., (2019)], and modeling an early warning system for dropout [Isiaka et al., (2019)]. Fig. 2 shows the adapted SEMMA methodology in this study.

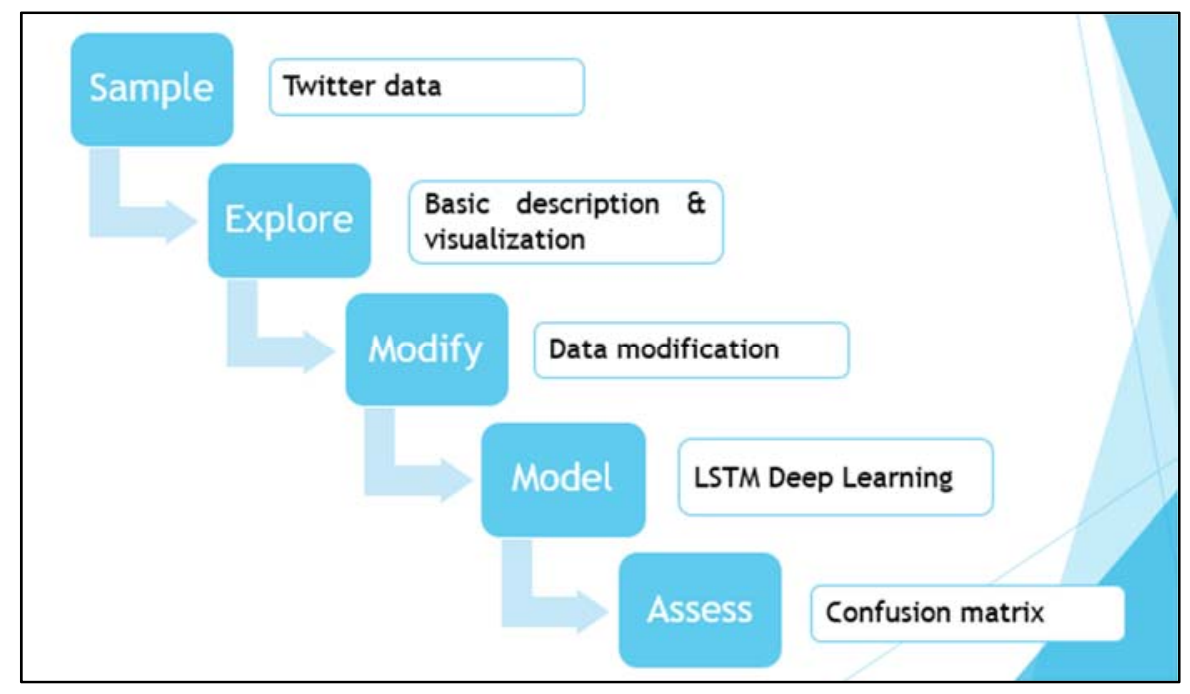

Fig. 2. SEMMA methodology in this study.

In the first step, we collected the tweets data sample in this study from the Twitter platform. We utilized the Tweepy library to access the Twitter API and collected tweets with the '\#PPKM' query from August $1^{\text {st }}, 2021$ to September $24^{\text {th }}, 2021$. There were 601 tweets successfully retrieved. Moreover, we used the TextBlob library to automatically classify each tweet's sentiment into three classes, i.e., positive class, negative class, and neutral class. The class proportion of collected tweets data is shown in Table 1. This collected data sample will be used for further analysis and classification using a deep learning method.

In the next step, we explored the collected tweets data by conducting some basic statistics description and data visualization. As can be seen from Fig. 3, the sentiment was dominated by neutral class, followed by positive class, and negative class. Moreover, we also tried to visualize several important words from each class by using the WordCloud library as shown in Fig. 4.

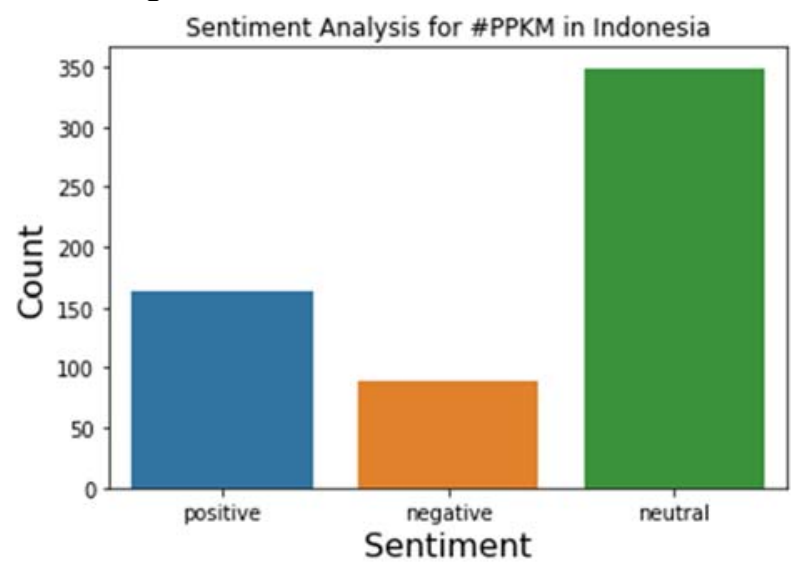

Fig. 3. Sentiment results using TextBlob library. 


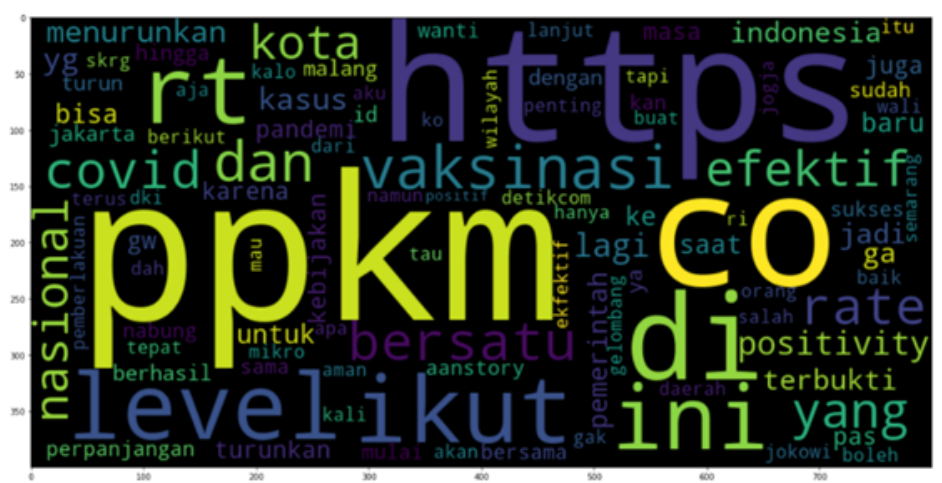

Fig.4. WordCloud visualization for the positive class.

In the third step, we modified the collected dataset so that it can be used in the data modeling phase. We built a new dataset that has a balanced number of data samples for each class. The new dataset was consisted of 267 tweets data, 89 tweets for each class, and will be used in the model development phase. Moreover, the new dataset was further modified to remove any punctuations and change the text case into lowercase. The new dataset was also tokenized to get 2,000 most frequent words found in the dataset.

In the model development phase, first, we split the dataset into training and test sets. We used an 80:20 ratio for the training and test sets, resulting in 213 training data and 54 test data. Next, we tried to apply LSTM networks with simple architecture. The deep networks consist of one embedding layer, one LSTM layer with 32 hidden neurons, and one dense layer with three output neurons - one for each target class in the dataset. Softmax activation function was used in this layer. Since this is a multi-categorical classification task, we used the categorical cross-entropy as the loss function during the model compilation optimized by the Adam optimizer.

Lastly, we evaluated the performance results of the proposed deep learning model by calculating the classification report to get precision, recall, and F1 score. We will also show the resulting confusion matrix and overall accuracy of the model tested on the test set. Further details will be given in the results section.

\section{Results and Discussion}

This section is divided into two sub-sections, the first one is dedicated to explaining the deep learning classification results and discussion, while the latter is dedicated to the deployment results of a simple web-based application for automated tweets classification.

\subsection{Deep learning classification results}

After we defined our deep learning architecture as explained in the previous section, we trained it on the training set. We determine the batch size for the training phase to be 32 and the number of epochs is 20 . The classification report and confusion matrix results of the trained model on the test set are shown in Fig. 5.

\begin{tabular}{|rrrrr|}
\hline [INF0] evaluating network... & & & \\
& precision & recall & f1-score & support \\
negative & 1.00 & 0.85 & 0.92 & 13 \\
neutral & 0.90 & 0.95 & 0.92 & 19 \\
positive & 0.91 & 0.95 & 0.93 & 22 \\
accuracy & & & & \\
macro avg & 0.94 & 0.92 & 0.92 & 54 \\
weighted avg & 0.93 & 0.93 & 0.93 & 54 \\
\hline
\end{tabular}

(a)

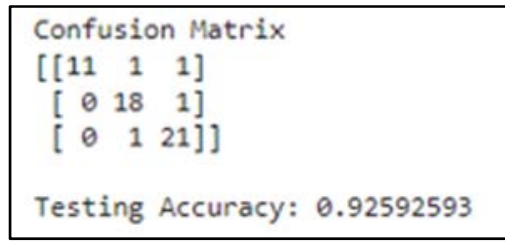

(b)

Fig. 5. Classification report (a) and confusion matrix results (b).

As can be seen from the report, the proposed LSTM model could successfully classify each tweet in the test set into its respective sentiment class. Out of 13 tweets in the negative class, 11 were correctly classified in the negative class, while two others were incorrectly classified in neutral and positive classes each. Moreover, out of 19 tweets in the neutral class, 18 of them were correctly classified in the neutral class and only one was incorrectly classified as the positive class. For the third class, out of 22 tweets in the positive class, 21 of them were correctly classified and only one was incorrectly classified as a neutral class. 
The overall accuracy result of the proposed model is $92.59 \%$, which is pretty high for this sentiment analysis classification task. The highest precision is on the negative class and the highest recalls are on both neutral and positive classes. However, when we used the F1 score metric, the positive class has the highest score at 0.93 meaning that among those three classes, the proposed deep learning model could predict the positive class slightly better than the other two classes in the dataset.

\subsection{Deployment results}

Using the built deep learning model, we tried to make a simple web-based application that can help in predicting new input tweets into positive, negative, and neutral classes. To do that, we built a flask application that takes the built model and tokenizer as the web-based application model. The application then will be deployed on the Heroku platform. Heroku is a cloud platform that enables developers to build, run, and operate applications quickly and easily [Salesforce.com, (2021)]. Fig. 6 shows the built web-based application and some examples of the automatic sentiment analysis results for the input tweets data. The application can be accessed freely on https://ppkm-analysis.herokuapp.com/.

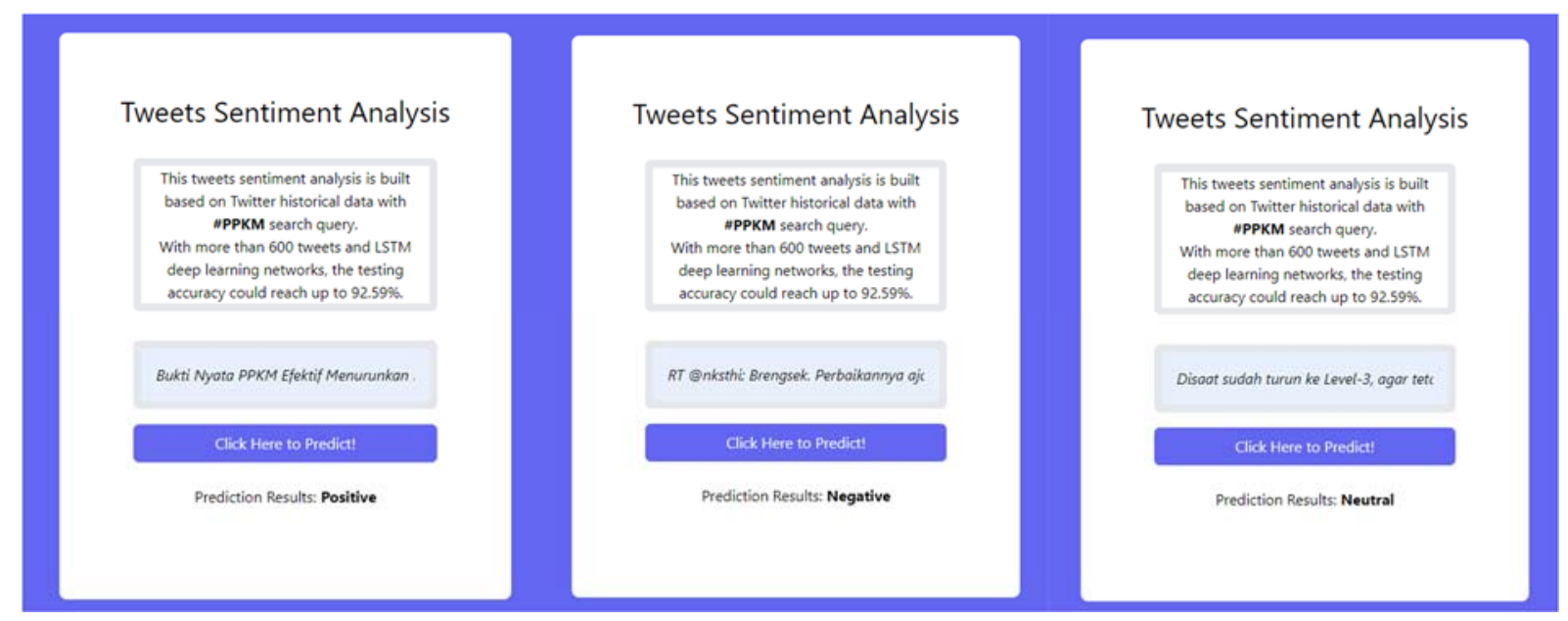

Fig.6. Simple web-based application for predicting tweets data.

\section{Conclusion}

We have successfully conducted the sentiment analysis for the PPKM policy in Indonesia. Using collected Twitter tweets data from August $1^{\text {st }}$, 2021 to September $24^{\text {th }}, 2021$, we found that most of the tweets can be classified as neutral sentiment (58.07\%), followed by positive sentiment $(27.12 \%)$, and lastly the negative sentiment (14.81\%). Therefore, it can be concluded that despite some negative impacts and ongoing restlessness in society, the PPKM policy could be accepted by most Indonesian people.

Moreover, we also conducted tweets classification using LSTM networks, a popular deep learning method for sentiment analysis tasks. We found that the accuracy results of the built model, which was trained on $80 \%$ of the collected tweets, could reach $92.59 \%$. Negative tweets sentiment has the highest precision, but positive tweets sentiment has the highest F1 score.

There are several limitations of this study that can be noticed and taken for future improvement. Firstly, we utilized the TextBlob library to conduct an automatic process for sentiment analysis of collected tweets data. By default, TextBlob uses a Naïve Bayes classifier on English text data. Since the collected tweets data is in Bahasa Indonesia, it would be better if the sentiment analysis process can be done manually or by considering the text pre-processing steps in Bahasa Indonesia. Another suggestion that can be made for future research is the use of several other machine learning, or even deep learning methods, in the classification task of the tweets data sentiments, such as Bayesian networks classifier [Ruz, et al., (2020)], Naïve Bayes, and Support Vector Machine [Hasan et al., (2018)], and deep Convolutional Neural Networks [Jianqiang, et al., (2018)].

\section{Acknowledgments}

We would like to acknowledge the support and facilitation given by Universitas Multimedia Nusantara during this study. The authors also would like to extend our acknowledge to all mentors in the Digital Talent Scholarship (DTS) program 2021 held by the Ministry of Communication and Information Technology of the Republic of Indonesia.

\section{References}

[1] Acter, T. et al. (2020): Evolution of severe acute respiratory syndrome coronavirus 2 (SARS-CoV-2) as coronavirus disease 2019 (COVID-19) pandemic: A global health emergency. Science of The Total Environment, 730, pp. 138996. doi: 
10.1016/j.scitotenv.2020.138996.

[2] Arora, R. et al. (2019): A Semi-Markov Structured Support Vector Machine Model for High-Precision Named Entity Recognition. Proceedings of the 57th Annual Meeting of the Association for Computational Linguistics. Stroudsburg, PA, USA: Association for Computational Linguistics, pp. 5862-5866. doi: 10.18653/v1/P19-1587.

[3] Asyary, A. and Veruswati, M. (2020): Sunlight exposure increased Covid-19 recovery rates: A study in the central pandemic area of Indonesia. Science of The Total Environment, 729, pp. 139016. doi: 10.1016/j.scitotenv.2020.139016.

[4] Djalante, R. et al. (2020): Review and analysis of current responses to COVID-19 in Indonesia: Period of January to March 2020. Progress in Disaster Science, 6, pp. 100091. doi: 10.1016/j.pdisas.2020.100091.

[5] Ferdina, V., Kristanda, M. B. and Hansun, S. (2019): Automated Complaints Classification using Modified Nazief-Adriani Stemming Algorithm and Naive Bayes Classifier. Journal of Theoretical and Applied Information Technology, 97(5), pp. 1604-1614.

[6] Fitri, V. A., Andreswari, R. and Hasibuan, M. A. (2019): Sentiment Analysis of Social Media Twitter with Case of Anti-LGBT Campaign in Indonesia using Naïve Bayes, Decision Tree, and Random Forest Algorithm. Procedia Computer Science, 161, pp. 765772. doi: 10.1016/j.procs.2019.11.181.

[7] Ginting, A. M. (2021): Optimism of Positive Economic Growth in 2021 in the Middle of Covid-19 Pandemic. Info Singkat, 13(1), pp. 19-24. Available at: https://berkas.dpr.go.id/puslit/files/info singkat/Info Singkat-XIII-1-I-P3DI-Januari-2021-224-EN.pdf.

[8] Hansun, S. and Suryadibrata, A. (2021): Gold Price Prediction in COVID-19 Era. International Journal of Computational Intelligence in Control, 13(2). Available at: https://www.mukpublications.com/ijcic-v13-2-2021.php.

[9] Hansun, S. and Young, J. C. (2021): Predicting LQ45 Financial Sector Indices using RNN-LSTM. Journal of Big Data, 8(1), pp. 104. doi: 10.1186/s40537-021-00495-x.

[10] Hasan, A. et al. (2018): Machine Learning-Based Sentiment Analysis for Twitter Accounts. Mathematical and Computational Applications, 23(1), pp. 11. doi: 10.3390/mca23010011.

[11] Jianqiang, Z., Xiaolin, G. and Xuejun, Z. (2018): Deep Convolution Neural Networks for Twitter Sentiment Analysis. IEEE Access, 6, pp. 23253-23260. doi: 10.1109/ACCESS.2017.2776930.

[12] Kennedy, P. S. J. (2021): Evaluation of the COVID-19 Handling Programs and National Economic Recovery in Indonesia. Fundamental Management Journal, 6(1), pp. 21-33. Available at: http://ejournal.uki.ac.id/index.php/jm/article/view/2831.

[13] Li, S. and Zhao, X. (2019): Image-Based Concrete Crack Detection Using Convolutional Neural Network and Exhaustive Search Technique. Advances in Civil Engineering, pp. 1-12. doi: 10.1155/2019/6520620.

[14] López-Torres, S. et al. (2020): IoT Monitoring of Water Consumption for Irrigation Systems Using SEMMA Methodology. Lecture Notes in Computer Science, Vol. 11886. Springer, Cham, pp. 222-234. doi: 10.1007/978-3-030-44689-5_20.

[15] Loria, S. (2020): TextBlob: Simplified Text Processing. Available at: https://textblob.readthedocs.io/en/dev/ (Accessed: 23 October 2021).

[16] Muhyiddin and Nugroho, H. (2021): A Year of Covid-19: A Long Road to Recovery and Acceleration of Indonesia's Development. The Indonesian Journal of Development Planning, 5(1), pp. 1-19. Available at: https://journal.bappenas.go.id/index.php/jpp/article/view/181.

[17] Isiaka, R.M. et al. (2019): A Machine Learning Approach to Dropout Early Warning System Modeling. International Journal of Advanced Studies in Computers, Science and Engineering, 8(2), pp. 1-12. Available at: https://www.proquest.com/docview/2198409061/fulltextPDF/DFC4B657F44F4281PQ/1?accountid=12763.

[18] Rahim, M. S., Rahman, M. and Chowdhury, A. E. (2017): Mining Industrial Engineered Data of Apparel Industry: A Proposed Methodology. International Journal of Computer Applications, 161(7), pp. 1-7. Available at: https://www.ijcaonline.org/archives/volume161/number7/27157-2017913262.

[19] Ribeiro, M. et al. (2018): Characterizing and Detecting Hateful Users on Twitter. Proceedings of the International AAAI Conference on Web and Social Media. Palo Alto, California USA, pp. 676-679. Available at: https://ojs.aaai.org/index.php/ICWSM/article/view/15057.

[20] Rufai, S. R. and Bunce, C. (2020): World leaders' usage of Twitter in response to the COVID-19 pandemic: a content analysis. Journal of Public Health, 42(3), pp. 510-516. doi: 10.1093/pubmed/fdaa049.

[21] Ruz, G. A., Henríquez, P. A. and Mascareño, A. (2020): Sentiment analysis of Twitter data during critical events through Bayesian networks classifiers. Future Generation Computer Systems, 106, pp. 92-104. doi: 10.1016/j.future.2020.01.005.

[22] Salesforce.com (2021): What is Heroku? Available at: https://www.heroku.com/what (Accessed: 24 October 2021).

[23] Spinelli, A. and Pellino, G. (2020): COVID-19 pandemic: perspectives on an unfolding crisis. British Journal of Surgery. doi: $10.1002 /$ bjs. 11627.

[24] Suhartono, D. et al. (2017): Personality Prediction Based on Twitter Information in Bahasa Indonesia. Proceedings of the Federated Conference on Computer Science and Information Systems. Prague, Czech Republic: IEEE, pp. 367-372. doi: 10.15439/2017F359.

[25] Tariq, H. I. et al. (2019): Loan Default Prediction Model Using Sample, Explore, Modify, Model, and Assess (SEMMA). Journal of Computational and Theoretical Nanoscience, 16(8), pp. 3489-3503. doi: 10.1166/jctn.2019.8313.

[26] Toharudin, T. et al. (2021): National Vaccination and Local Intervention Impacts on COVID-19 Cases. Sustainability, 13(15), pp. 8282. doi: $10.3390 /$ su13158282.

[27] Vistro, D. M., Rasheed, F. and David, L. G. (2019): The Cricket Winner Prediction with Application of Machine Learning and Data Analytics. International Journal of Scientific \& Technology Research, 8(9), pp. 985-990. Available at: https://www.kclas.ac.in/wpcontent/uploads/2021/01/The-Cricket-Winner-Prediction-With-Application-Of-Machine-Learning-And-Data-Analytics-.pdf.

[28] Walsh, S. (2021): The Top 10 Social Media Sites \& Platforms 2021, SEJ. Available at: https://www.searchenginejournal.com/socialmedia/biggest-social-media-sites/\#close (Accessed: 23 October 2021).

[29] World Health Organization (2021): WHO Coronavirus (COVID-19) Dashboard, WHO. Available at: https://covid19.who.int/ (Accessed: 23 October 2021).

[30] Yu, L. et al. (2019): Predicting Monthly Biofuel Production using a Hybrid Ensemble Forecasting Methodology. International Journal of Forecasting, In Press. doi: 10.1016/j.ijforecast.2019.08.014.

[31] Yunita, I. and Hansun, S. (2019): Automatic News Blog Classifier using Improved k-Nearest Neighbor and Term Frequency-Inverse Document Frequency. Journal of Theoretical and Applied Information Technology, 97(15), pp. 4202-4212. Available at: http://www.jatit.org/volumes/Vol97No15/16Vo197No15.pdf. 


\section{Authors Profile}

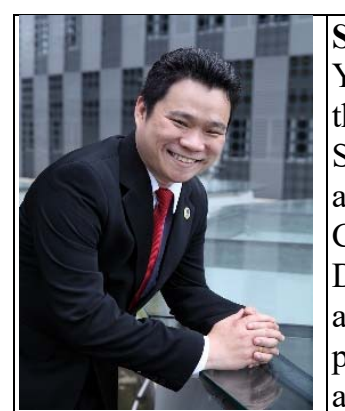

Seng Hansun received the Bc. degree in Mathematics from Universitas Gadjah Mada, Yogyakarta, in 2008 and Master of Computer Science degree in Computer Science from the same university in 2011. Since then, he has been a Lecturer with the Computer Science Department, Universitas Multimedia Nusantara (UMN), Indonesia. He had been appointed as the Information and Communication Technology (ICT) Faculty Research Coordinator, Deputy Head of Computer Science Department, and Head of Informatics Department at UMN. He has published two books, one in Discrete Mathematics and another in Mobile Android Programming, distributed widely in Indonesia. He also has published more than 135 articles during his career as both academician and researcher at UMN. He has served as a Technical Program Committee (TPC) member in several international conferences and acted as a Reviewer and Editorial Board of both national and international journals. His research interests lately include computational science, soft computing methods, and internet and mobile technology in various fields, especially in the Medical Informatics area.

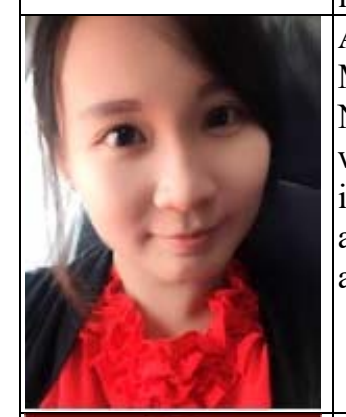

Alethea Suryadibrata is a lecturer at the Department of Informatics, Universitas Multimedia Nusantara, Indonesia. She was graduated from Universitas Multimedia Nusantara with Bachelor in Computer Science degree in 2014 and from Silla University with M.Eng degree in 2017. Her main teaching and research interests include digital image processing, computer graphics and animation, reliable software engineering, and applied artificial intelligence and machine learning. She has published several research articles in various international peer-reviewed journals.

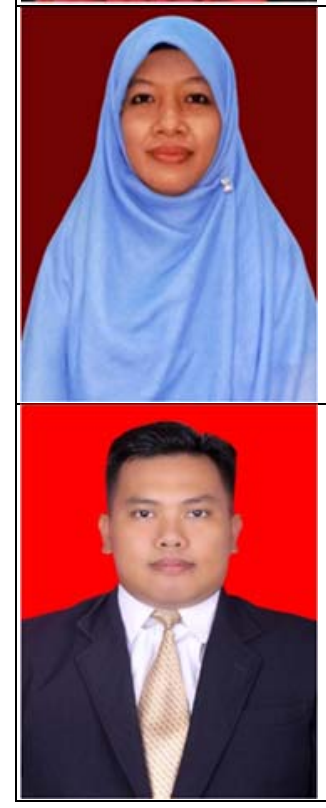

Rossy Nurhasanah is a senior lecturer at the Information Technology Department, Universitas Sumatera Utara, Indonesia. She received her Bachelor degree in Computer Science from Universitas Sumatera Utara in 2010 and Master degree in Computer Science from Institut Pertanian Bogor in 2015. She has lectured several core courses, such as Graph Theory and Application, Data Structure and Algorithm, and Discrete Mathematics. Her research interests include bioinformatics, machine learning, and computational intelligence.

Jaka Fitra is a lecturer at the Institut Teknologi dan Bisnis Diniyyah Lampung, Bandar Lampung, Indonesia. He was graduated from undergraduate program at Universitas Mitra Indonesia majoring Information System and from master program at Institut Informatika dan Bisnis (IIB) Darmajaya majoring Information Technology. His research interests are computer vision and applied information technology. 